\title{
Norois
}

Environnement, aménagement, société

$215 \mid 2010 / 2$

Impacts morphogéniques des tempêtes

\section{Effets des tempêtes sur une plage aménagée et à forte protection côtière : la plage des Éloux (côte de Noirmoutier, Vendée, France)}

Impacts of storms on a managed and protected shoreline: Les Éloux Beach (Noirmoutier coast, Vendée, France)

Paul Fattal, Marc Robin, Martin Paillart, Mohamed Maanan, Denis Mercier, Christine Lamberts et Stéphane Costa

\section{OpenEdition}

\section{Journals}

Édition électronique

URL : http://journals.openedition.org/norois/3289

DOI : $10.4000 /$ norois.3289

ISBN : 978-2-7535-1563-5

ISSN : $1760-8546$

Éditeur

Presses universitaires de Rennes

Édition imprimée

Date de publication : 30 septembre 2010

Pagination : 101-114

ISBN : 978-2-7535-1164-4

ISSN : 0029-182X

Référence électronique

Paul Fattal, Marc Robin, Martin Paillart, Mohamed Maanan, Denis Mercier, Christine Lamberts et Stéphane Costa, «Effets des tempêtes sur une plage aménagée et à forte protection côtière : la plage des Éloux (côte de Noirmoutier, Vendée, France) », Norois [En ligne], 215 | 2010/2, mis en ligne le 01 septembre 2012, consulté le 01 mai 2019. URL : http://journals.openedition.org/norois/3289 ; DOI : 10.4000/norois.3289 


\title{
EFFETS des TEMPÊTES SUR UNE PLAGE AMÉNAGÉE ET À ForTe PRotection Côtière : LA PLAGE des ÉLOUX (Côte de Noirmoutier, Vendée, France)
}

\author{
Paul Fattal \\ Géolittomer - UMR 65554 LETG CNRS \\ (Université de Nantes),
}

Chemin de La Censive-du-Tertre, BP 81227 - 44312 Nantes Cedex 3, France

Paul.Fattal@univ-nantes.fr

Marc Robin

Géolittomer - UMR 6554 LETG CNRS

(Université de Nantes),

Chemin de La Censive-du-Tertre, BP 81227 - 44312 Nantes Cedex 3, France

Marc.Robin@univ-nantes.fr

\section{Martin Paillart}

Communauté de Communes de l'Ile de Noirmoutier

Prée aux Ducs - 85330 Noirmoutier-EN-L'ÎleE, France

m-paillart@wanadoo.fr

MOHAMEd MaAnaN

Géolittomer - UMR 6554 LETG CNRS

(Université de Nantes),

Chemin de La Censive-du-Tertre, BP 81227 - 44312 Nantes Cedex 3, France Mohamed.Maanan@univ-nantes.fr

Denis Mercier

Géolittomer - UMR 6554 LETG CNRS

(Université de Nantes),

Chemin de La Censive-du-Tertre, BP 81227 - 44312 Nantes Cedex 3, France Denis.Mercier@univ-nantes.fr

\section{Christine Lamberts}

(Géolittomer - UMR 6554 LETG),

Chemin de La Censive-du-Tertre, BP 81227 - 44312 Nantes Cedex 3, France Christine.Lamberts@univ-nantes.fr

\section{Stéphane Costa}

Géophen - UMR 6554 LETG CNRS

(Université de Caen - Basse-Normandie),

Esplanade de la Paix, BP 5186 - 14032 CaEn Cedex, France

stephane.costa@unicaen.fr 


\section{RÉSUMÉ}

L'analyse diachronique des données disponibles sur le long terme montre que l'érosion de la plage des Éloux dans l'île de Noirmoutier (France) s'est produite entre 1832 et 2008 à des vitesses variables avec un taux moyen de recul du trait de côte de $0,7 \mathrm{~m} / \mathrm{an}$. La période 1999-2008 enregistre un fort recul du trait de côte avec un taux moyen de 1,9 m/an. Dans ce contexte, une forte tempête comme celle du 10 mars 2008, si elle contribue à la tendance érosive générale, n'a pas entraîné de conséquences morphogéniques très importantes sur l'île de Noirmoutier. Cet article cherche à estimer cet impact sur la plage des Éloux et à mettre en lumière le fait que l'effet minime de cette tempête importante est imputable à divers facteurs qui sont discutés (dérive inversée, stock sédimentaire disponible sur l'avant plage, défenses côtières).

MOTS CLÉ : tempête - plage - érosion - aménagement littoral

\section{ABSTRACT \\ Impacts of storms on a managed and protected shoreline : Les Éloux Beach (Noirmoutier coast, Vendée, France)}

The diachronic analysis of available data shows that the erosion of the Eloux beach along Noirmoutier Island occurred at variable rates between 1832 and 2008 with a mean rate of 0,7 m/year. The period between 1997-2008 shows generalized erosion with a mean rate of 1,9 m/year. The impact of the 10 Marsh 2008 storm on the coastline of Noirmoutier Island appears moderate compared to these rates. This paper aims at assessing the impact of this storm on Les Éloux Beach. It is believed that the relatively low impact of this storm is mainly due to three factors : reversed littoral drift, intertidal sand bank source, and coastal defence.

KEY WORDS : storm - beach - erosion - coastal management

L'île de Noirmoutier, de par sa situation, est très exposée aux tempêtes (Gautier, 1972, 1979). En raison des considérables enjeux de nature et de société caractérisant le milieu insulaire, le suivi de l'impact des tempêtes revêt ici une dimension essentielle et justifie l'actuelle mise en place d'un Plan de Prévention des Risques d'Inondation. Globalement, l'intensité des impacts des tempêtes sur les plages sableuses dépend de facteurs physiques et socio-économiques (Battiau-Queney et al., 2003; Regnauld et al., 2004). Ces événements extrêmes, favorisés par l'effet des changements climatiques (Wakelin et al., 2003), se produisent lors du passage d'une forte perturbation atmosphérique responsable de l'apparition de vents violents d'afflux qui poussent la masse d'eau vers les côtes, ce qui, combiné aux houles, entraîne la formation de surcotes. Celles-ci deviennent extrêmes lorsqu'il y a coïncidence avec une marée haute et un fort coefficient de marée (Bouligand et Pirazzoli, 1999; Pirazzoli et al., 2006; Pirazzoli et al., 2007; Caspar et al., 2007).

Le 10 mars 2008, une tempête de cette nature a frappé les côtes du Nord-Ouest de la France. L'île de Noirmoutier au sud de l'estuaire de la Loire, n'est pas épargnée par cet épisode atmosphérique de forte intensité, même si celui-ci n'est pas exceptionnel pour cet espace insulaire. En effet, les rafales n'ont pas dépassé les $95 \mathrm{~km} / \mathrm{h}$ alors qu'elles ont dépassé les $100 \mathrm{~km} / \mathrm{h}$ à plusieurs reprises depuis les années 1960, allant jusqu'à $135 \mathrm{~km} / \mathrm{h}$ lors de la tempête des 12 et 13 septembre 1993 (Tonnerre, 2001). Les secteurs de l'île qui ont été affectés par cette tempête sont en majorité en érosion et sont dotés de fortes défenses contre la mer (CCN, 2000). 


\section{Caractéristiques de la zone d'étude}

\section{CADRE MORPHOLOGIQUe gÉNÉRAL}

L'île de Noirmoutier s'est progressivement formée depuis la fin de la dernière période froide du Pléistocène. La présence d'une plateforme calcaire reposant sur un socle cristallin affleurant dans la partie nord de l'île a permis le piégeage des sables en provenance de la Loire ou de la plateforme continentale (Morzadec-Kerfourn, 1995). Cette plateforme d'érosion littorale se manifeste par des points hauts depuis les écueils les plus à l'ouest (les Bœufs au large de la pointe du Devin) jusqu'aux écueils les plus à l'est (la Vendette) et depuis le nord (les Pères) jusqu'au sud (la Fosse) (fig. 1). Depuis 4000 ans, la translation de la couverture sédimentaire s'est effectuée depuis l'ouest (recouvrant alors les Bœufs $4 \mathrm{~km}$ à l'ouest de l'emplacement actuel du cordon dunaire des Éloux) vers l'est, découvrant successivement les divers pointements rocheux (Ters, 1961). La situation actuelle correspond à un cordon ancré sur le récif du Devin au nord et le récif de la Loire au sud.

L'île de Noirmoutier est une île basse (altitude maximum de $22 \mathrm{~m}$ ) de $20 \mathrm{~km}$ de longueur pour $6 \mathrm{~km}$ de largeur maximale dont les $2 / 3$ de la surface sont situés sous le niveau des pleines-mers de vive-eau (fig. 1).

Figure 1 : La zone d'étude Study area

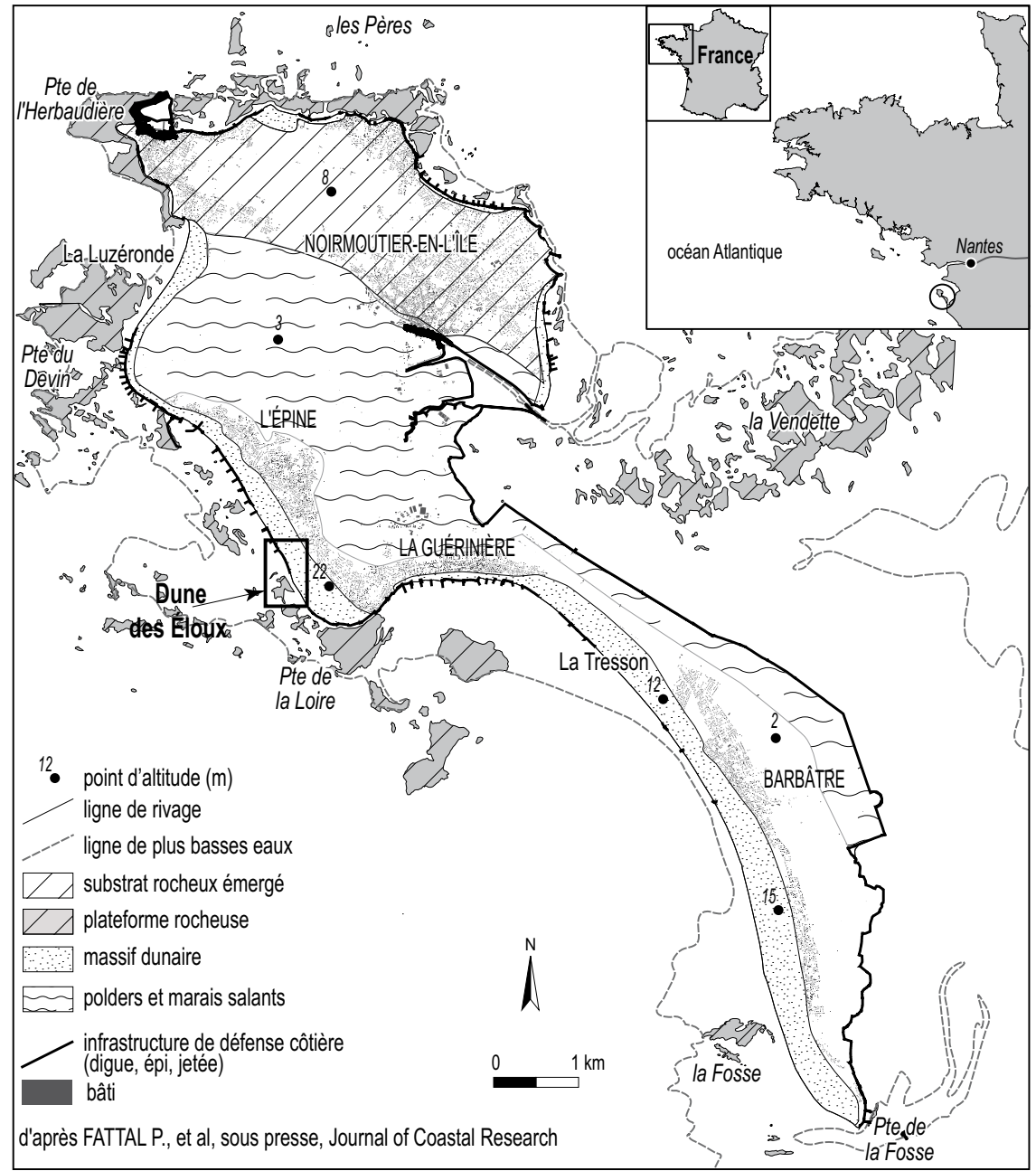


La zone infralittorale est faiblement pentue : l'isobathe - $5 \mathrm{~m}$ (cote marine) est située en moyenne à $3 \mathrm{~km}$ au large vers l'ouest ce qui lui confère une pente inférieure à $0,16 \%$. Côté baie de Bourgneuf à l'est, du fait de la position d'abri, la pente infralittorale est encore plus faible. La côte occidentale de l'île est subdivisée en 3 cellules hydrosédimentaires encadrées successivement par les pointes de L'Herbaudière, du Devin, de la Loire et le goulet de Fromentine (pointe de la Fosse) (DHI, 2008). De L'Herbaudière au Devin s'étend la cellule de Luzéronde; du Devin à la pointe de la Loire se situe celle des Éloux qui fait l'objet de cette évaluation; de la pointe de la Loire à la pointe de la Fosse au sud se développe la cellule de La Guérinière/Barbâtre. L'orientation générale moyenne de ces 3 segments côtiers varie : de l'ordre de $210^{\circ}$ pour Luzéronde, elle est de $157^{\circ}$ pour les Éloux, et de $160^{\circ}$ pour Barbâtre.

Le segment des Éloux s'étend sur 4,5 km de long sur la commune de l'Épine. Il est bordé à l'arrière par un massif dunaire qui s'élargit sur près de $500 \mathrm{~m}$ et culmine à $22 \mathrm{~m}$. Ce secteur est fortement protégé (photo 1). La dérive littorale dominante résiduelle attestée par le piégeage des sédiments par les épis se fait du nord vers le sud. Le transit sédimentaire longitudinal moyen est évalué à $20000 \mathrm{~m}^{3} / \mathrm{an}$ (DHI, 2008).

L'analyse granulométrique du sable de la plage montre qu'il est grossier et mal classé : médiane $=1$, Sorting index $=2-$ valeurs exprimées en Phi (Folk, 1966), dû en partie, comme nous le verrons ultérieurement, au fait qu'il y a eu des rechargements avec des sédiments hétérométriques en provenance de la pointe de la Fosse.

\section{CaractÉristiques mÉtÉo-MARINES gÉNÉRALES}

De façon générale, à Noirmoutier, les vents de secteur nord-ouest dominent et représentent $26,2 \%$ des occurrences. Viennent ensuite les vents de nord-est $(24,2 \%)$ et ceux de sud-ouest $(22,6 \%)$. Les vents de vitesses supérieures à $8 \mathrm{~m} / \mathrm{s}$ (supérieurs à force 5 Beaufort) représentent globalement $8,1 \%$ des observations. $86,4 \%$ de ces vents proviennent du secteur sud-ouest à nordouest (Météo France, station de Noirmoutier-en-l'Île).

L'interprétation des données de houles fournies par la base de données de l'atlas numérique d'états de mer océanique et côtier (ANEMOC) du CETMEF permet de cerner leur caractéristique le long de la côte noirmoutrine : les houles les plus fréquentes $(-40 \%)$ ont une amplitude comprise entre 1,25 m et 2,5 m et proviennent du secteur ouest à nord-ouest. En revanche, les houles de plus forte amplitude (supérieures à $6 \mathrm{~m}$ ) proviennent du secteur ouest à sud-ouest.

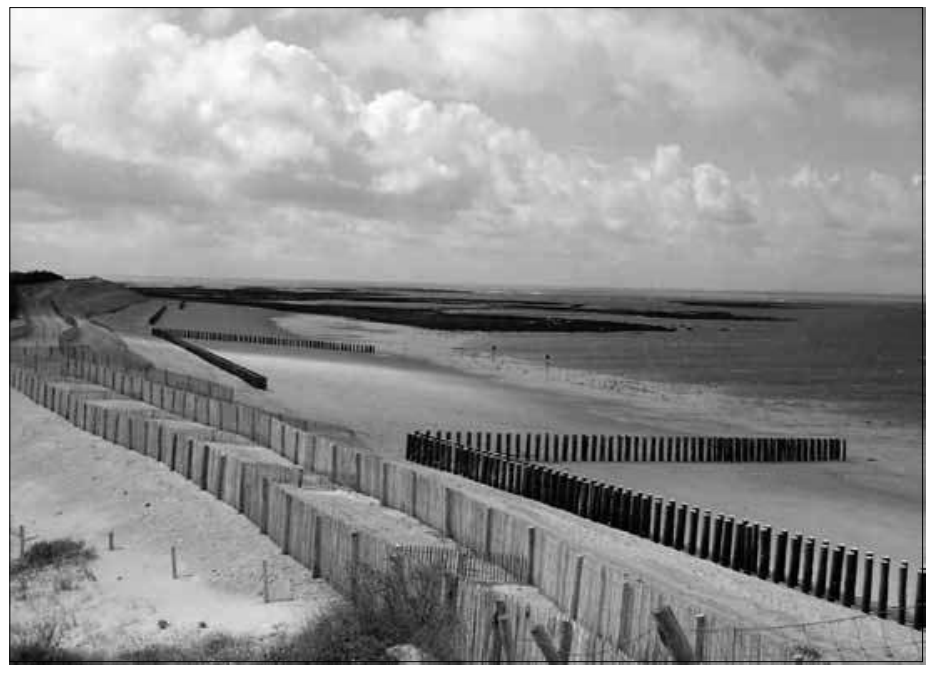

Photo 1 : Protections côtières dans le secteur des Éloux (2007) Shoreline defence structures along Les Eloux beach 


\section{UN SECTEUR À IMPORTANTE PROTECTION CÔTIÈRE}

Depuis plus d'un siècle, la côte ouest de Noirmoutier a été et est soumise à des phénomènes importants d'érosion du trait de côte conduisant les habitants de l'île à engager des actions de protection contre la mer, dès 1770 (au niveau du Devin). La plage des Éloux est, elle aussi, soumise à une érosion qui explique les nombreux aménagements de protection côtière (fig. 2).
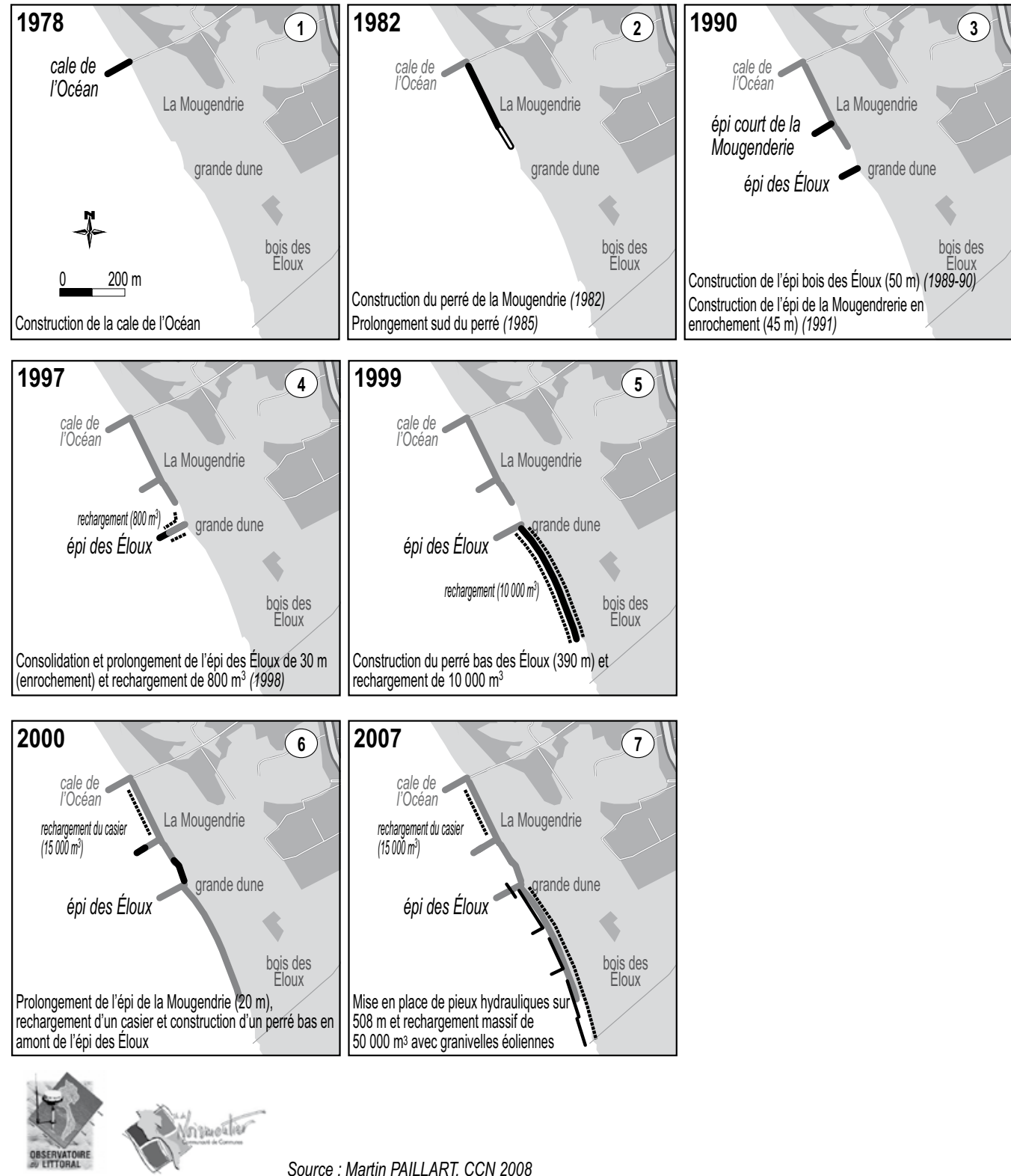

Source : Martin PAILLART, CCN 2008

Figure 2 : Les aménagements de protection côtière dans le secteur des Éloux Coastal defence structures along Les Éloux beach 
En raison d'un recul local parfois important (4 m entre septembre 2004 et novembre 2004), la communauté de communes de l'île a entrepris plusieurs types de travaux.

En effet, entre décembre 2006 et avril 2007 un rechargement a été effectué et $51000 \mathrm{~m}^{3}$ de sédiments en provenance de la pointe de la Fosse ont été ramenés sur la plage (fig. 1). Ce rechargement a par ailleurs été complété par de nouveaux apports réalisés entre octobre et novembre 2007, puis après la tempête de mars 2008 (tableau 1). Au total, le volume du rechargement est de l'ordre de $54000 \mathrm{~m}^{3}$ et il ne compense que partiellement les pertes observées entre 1999 et 2007 qui ont été évaluées à plus de $100000 \mathrm{~m}^{3}$ (CCN, 2008).

Par ailleurs, la communauté de communes de l'île a mis en place des ganivelles sur le massif dunaire reconstitué et reprofilé et, enfin, des pieux hydrauliques (février à juin 2007) ont été positionnés sur la plage. Ces deux derniers aménagements (photo 1) s'étendent sur une longueur de $500 \mathrm{~m}$ et sont tous deux destinés, à travers l'atténuation désirée de l'énergie des houles et du vent, à ralentir l'érosion éolienne et marine.

\begin{tabular}{|c|c|c|}
\cline { 2 - 3 } \multicolumn{1}{c|}{} & Dates & Volumes rechargés $(\mathrm{m} 3)$ \\
\hline 1 & décembre 2006 à avril 2007 & 51000 \\
\hline 2 & octobre 2007 & 570 \\
\hline 3 & novembre 2007 & 1200 \\
\hline 4 & avril 2008 & 2000 \\
\hline & total & 54770 \\
\hline
\end{tabular}

Tableau 1 : Inventaires des rechargements récents de la plage des Éloux

Inventory of recent beach nourishment of Les Éloux beach

\section{Méthodologie de suivi}

La méthodologie repose sur l'exploitation classique de deux sources d'information : l'iconographie d'un côté et les levés de terrain de l'autre.

Une étude diachronique précise du trait de côte a d'abord été menée à l'aide de photographies aériennes couvrant une période de 56 ans étendue à une période de 174 ans en y ajoutant l'exploitation géométrique du cadastre napoléonien. Une base de données image a ainsi été constituée après numérisation et géoréférencement. La ligne de référence digitalisée est le contact dune/ plage (Robin, 2002). La comparaison en 2D de ces lignes renseigne la dimension surfacique de l'évolution.

Dans un second temps, les levés de plage effectués saisonnièrement, depuis la fin des années 1990, lors des marées de vives-eaux, au moyen d'un DGPS (DGPS TRIMBLE 4400 et 4700 précision de $1 \mathrm{~cm}$ en $\mathrm{X}$, Y et $3 \mathrm{~cm}$ en Z) ont été exploités. Ces relevés d'environ 5000 points permettent de produire des Modèles Numériques de Terrains comparables entre eux, et de calculer l'évolution volumétrique du bilan sédimentaire. Les bilans sédimentaires calculés pour les cellules hydrosédimentaires concernent des périodes comprises entre 1999 et 2009 . Pour le secteur d'étude la période est comprise entre juin 2006 et mars 2009 (tableau 3). En effet, plusieurs campagnes ont été réalisées dans le cadre de l'observatoire du littoral de l'île de Noirmoutier (2006 : juin; 2007 : avril, juin, septembre, décembre; 2008 : mars, juin, septembre, décembre; 2009 : mars). Enfin, un levé de l'avant plage a aussi été effectué en 2006 au droit des Eloux jusqu’à -5 m (cote marine). 


\section{Résultats}

\section{ÉVOLUTIONS SUR LE LONG TERME}

Depuis 1832, on observe des reculs du rivage sur toute la façade exposée de l'île de Noirmoutier, jusqu'au droit de la commune de Barbâtre. De là et jusqu'à la pointe de la Fosse, le rivage gagne sur la mer (tableau 2). Ces reculs du rivage sont compris entre 100 et $150 \mathrm{~m}$ pour le secteur situé entre l'Épine et la pointe de la Loire et sont de l'ordre de $120 \mathrm{~m}$ pour la plage des Éloux (fig. 3). Au contraire, au sud de l'île, du fait du transfert latéral des sédiments, on observe une progradation de l'ordre de $130 \mathrm{~m}$ au droit de la commune de la Barbâtre atteignant $320 \mathrm{~m}$ à la pointe de la Fosse (Observatoire du littoral de l'île de Noirmoutier - communauté de communes de l'île que l'on nommera CCN, 2000).
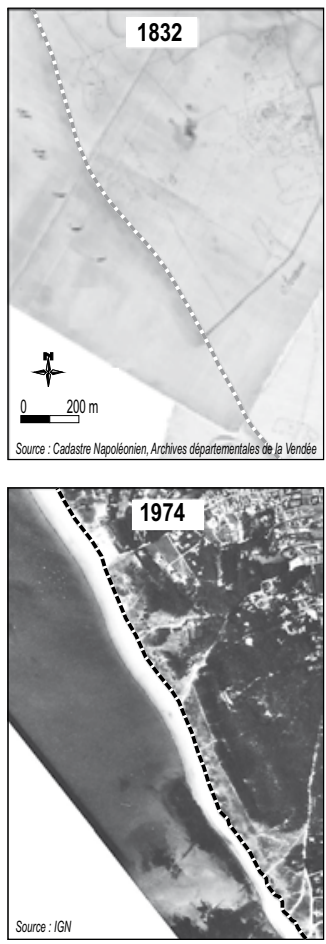
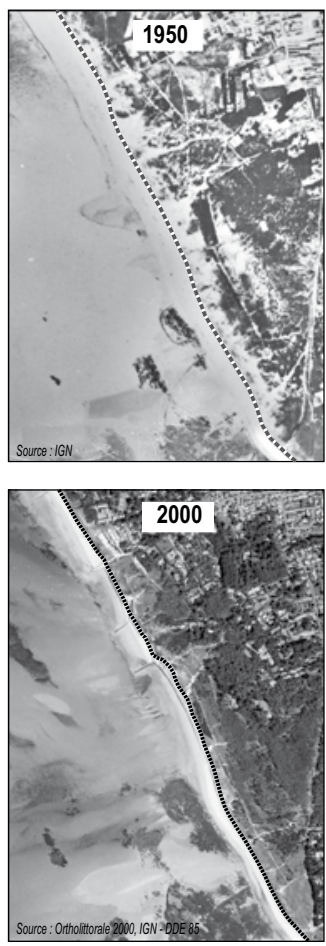

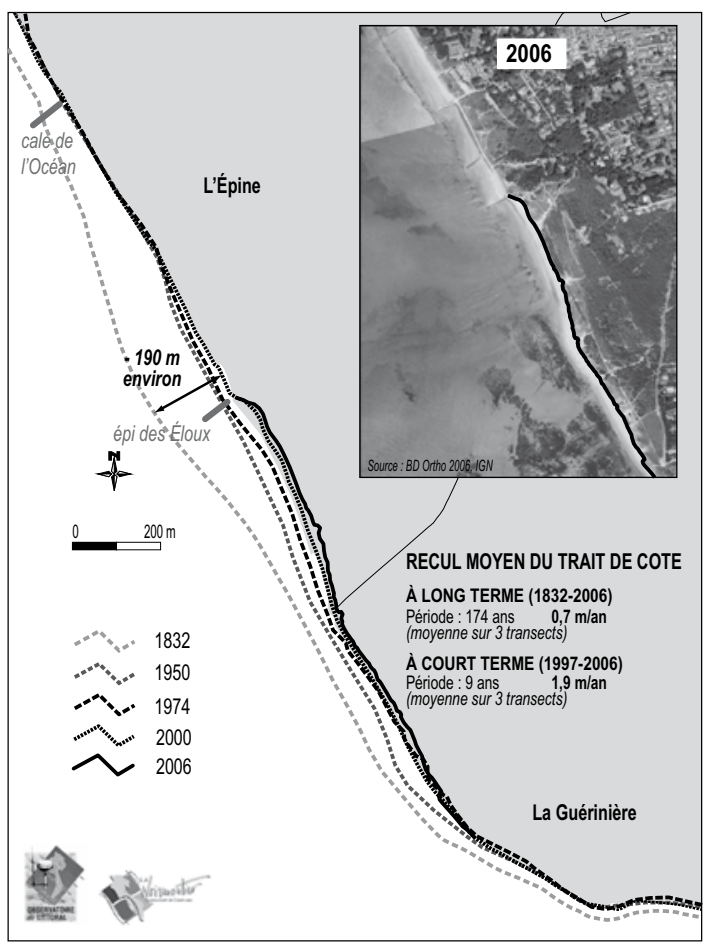

Figure 3 : Cinématique du trait de côte de la plage des Éloux depuis 1832 (zone encadrée sur la figure 1) Shoreline change of the Eloux beach since 1832

Cette érosion récurrente au droit des Éloux a engendré comme réponse, la mise en place de toute une batterie de mesures de protections en «dur » ainsi que des rechargements de la plage et de la dune (fig. 2). La construction de la cale de l'océan en 1978 et la succession d'aménagements de protections (perrés, épis, etc.) n’ont toutefois pas empêché cette érosion. Le recul au niveau des Éloux est de l'ordre de 0,7 m/an entre 1832 et 2006. Il atteint 1,9 m/an entre 1999 et 2006. Dans ce secteur, le volume perdu (hors rechargements) entre octobre 1999 et le début de l'année 2007 est de 103700 m $^{3}$ (CCN, 2008). 


\begin{tabular}{|c|l|c|}
\hline Cellules hydrosédimentaires & \multicolumn{1}{|c|}{ Période 1999-2007 } & bilans sédimentaires en $\mathrm{m}^{3}$ \\
\hline Pointe de L'Herbaudière/Pointe du Devin & plage de la Luzeronde & +17400 \\
\hline \multirow{3}{*}{ Pointe du Devin/Pointe de la Loire } & plage du Morin & +88900 \\
\cline { 2 - 3 } & plage de l'Epine & -68500 \\
\cline { 2 - 3 } & plage des Eloux & -103700 \\
\hline \multirow{3}{*}{ Pointe de la Loire/Pointe de la Fosse } & plage de la Guérinière & -700 \\
\cline { 2 - 3 } & plage de la Tresson & +61300 \\
\cline { 2 - 3 } & Pointe de la Fosse & +100000 \\
\hline
\end{tabular}

Tableau 2 : Bilans sédimentaires par cellules hydrosédimentaires (source : CCN, 2008) Sediment budget for each sediment cells

\section{ÉVOLUTION SUR LES COURT ET MOYEN TERMES}

Caractéristiques météo-marines pendant la tempête de mars 2008

La tempête du 10 mars 2008 s'est accompagnée de vents moyens de $70 \mathrm{~km} / \mathrm{h}$ avec des rafales enregistrées à $95 \mathrm{~km} / \mathrm{h}$ à la station de Noirmoutier-en-l'Île. Leur provenance était majoritairement de secteur sud-ouest $\left(200^{\circ}\right.$ en moyenne le 10 mars avec des rafales notées à $210^{\circ}$, contre un vent moyen à $50 \mathrm{~km} / \mathrm{h}$ le 11 mars de direction $280^{\circ}$, avec des rafales enregistrées à $72 \mathrm{~km} / \mathrm{h}$ de direction $300^{\circ}$ ). Pendant cet épisode, la dérive littorale était orientée sud-nord, inverse donc à la direction résiduelle attestée par le piégeage des sédiments dans les ouvrages de protection (voir discussion). Cette dynamique hydrosédimentaire induite par la tempête n'a pas été particulièrement originale. En effet, M. Gautier (1972) a montré que lors des tempêtes ayant affecté la zone d'étude entre 1959 et 1967, les vents avaient soufflé dans $54 \%$ des cas du quart sud-ouest, dans $33 \%$ du quart nord-ouest et seulement dans $8,5 \%$ et $4,5 \%$ des quarts ouest et nord-est, alors que toute l'année ce sont plutôt les vents de nord-ouest qui dominent.

L'autre caractéristique de cet évènement vient de la coïncidence entre de très forts coefficients de marée (106), une pleine mer prédite à + 5,5 m à 6 h $09(\mathrm{UT}+1)$ le 10 mars (fig. 4) et une pression barométrique qui a atteint son point le plus bas à $985 \mathrm{hPa}$ entre 6 heures et 7 heures le 10 mars. Ces surcotes, se produisant durant l'hiver, principalement d'octobre à mars, deviennent géomorphologiquement actives dans les processus d'érosion du littoral (Sabatier et al., 2009). La surcote barométrique enregistrée au port de Saint-Nazaire, a été de + 1,3 m (hauteurs d'eau 6,8 m, enregistrement SONEL), soit $+40 \mathrm{~cm}$ liée à la dépression barométrique, le reste étant la surcote dynamique liée au vent (il est à noter que l'effet de l'estuaire et du débit de la Loire est de nature à gonfler la surcote à Saint-Nazaire). De ce fait, la surcote mesurée aux Sables-d'Olonne a donc été moindre (hauteur d'eau à la même heure : + 6,05 m) (fig. 4)

On peut donc estimer que la surcote qui a dû se produire à 6 heures au niveau des Éloux devait être de l'ordre de celle des Sables-d'Olonne soit une hauteur d'eau d'environ 0,5 à 0,6 m au dessus de la hauteur d'eau prédite. Cette estimation ne tient pas compte de l'augmentation locale du plan d'eau (wave set-up), induit par le déferlement au niveau de la plage (Longuet-Higgins et Stewart, 1964; Svendsen, 1984; Greenwood et Osborne, 1990; Gourlay, 1992 ; Swift, 1993). Malheureusement, la bouée d'enregistrement des houles de l'île d'Yeu à $25 \mathrm{~km}$ au sud-ouest des Eloux n'a pas fonctionné durant la journée du 10 mars ce qui n’a pas permis une estimation plus précise de cette composante dans la surcote. Le dernier enregistrement de la bouée est du 9 mars 2008 à 18 heures : le $\mathrm{H}_{\max }$ était alors de $3,47 \mathrm{~m}$ pour une période de $8,9 \mathrm{~s}$ tandis que le $\mathrm{H}_{1 / 3}$ était de 2,11 $\mathrm{m}$ avec une période de 8 secondes.

Au total, cette conjonction de paramètres susceptibles d'amplifier l'érosion, lors de cet épisode du 10 mars 2008, reste originale même si elle n'est pas a priori exceptionnelle. En effet, lors de la 
Figure 4 : Hauteur d'eau prédite $(\mathrm{mP})$ et hauteur d'eau observée $(\mathrm{mO})$ dans le port des Sables-d'Olonne heure par heure (de Oh UT le 10 mars à 23 heures UT le 11 mars 2008) Predicted water level $(\mathrm{mP})$ and observed water level (Mo) in the harbour of Les Sables-d'Olonne

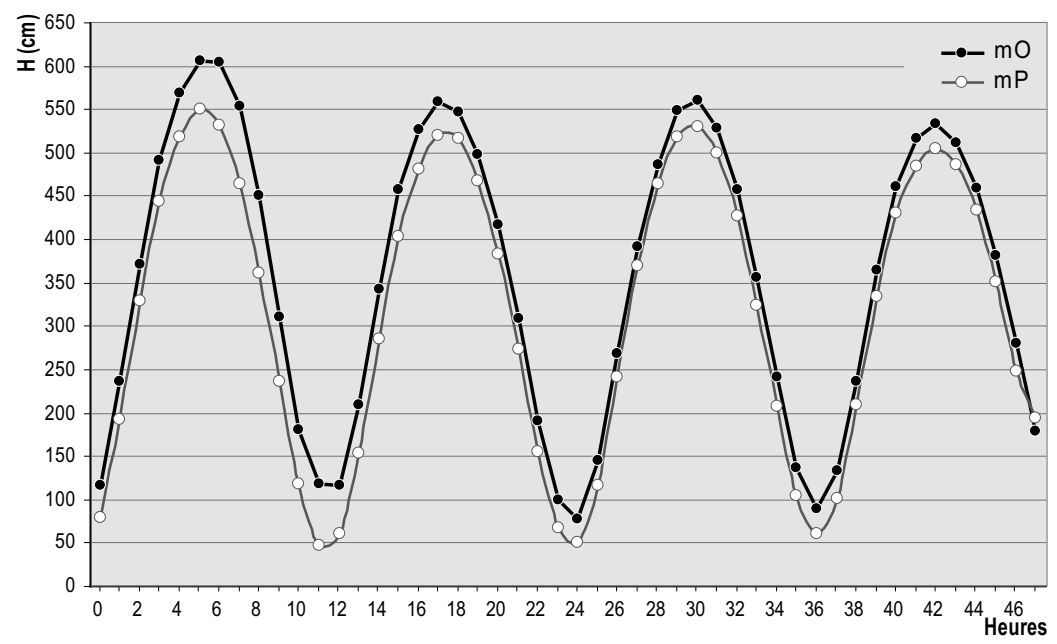

tempête de fin décembre 1978 et début janvier 1979, les coefficients de marée avaient également atteint 102 (le 31) et 104 (le premier janvier) (Gautier, 1979).

\section{Le bilan sédimentaire de la tempête de mars 2008 par rapport à un bilan à moyen terme}

Entre décembre 2007, c'est-à-dire après le rechargement, et mars 2008, la plage a perdu près de $4900 \mathrm{~m}^{3}$ dont une partie peut être imputée à la tempête du 10 mars 2008 (tableau 3 et fig. 6). En effet, après la tempête, les reculs du trait de côte ont été très importants au nord de la plage puisqu'ils ont été localement plus de 12 m (fig. 5). En comparaison, lors de la tempête de 2004 (novembre), le recul maximal a été, sur le même secteur, inférieur à $5 \mathrm{~m}$. Il faut remonter à 2003, après la tempête de janvier, pour observer un recul aussi important du trait de côte : plus de $10 \mathrm{~m}$ entre l'arrière du perré des Éloux et les enrochements de la pointe de la Loire (CCN, 2004). Enfin, l'année 1999 est marquée par deux tempêtes, celles d'octobre et de décembre. Elles n'ont eu sur le secteur qu'un effet limité en raison de l'édification, en mars de la même année, d'un perré bas localisé au sud de l'épi des Éloux sur 390 m (aujourd'hui en grande partie disloqué). L'ouvrage a partiellement ralenti l'attaque de la dune qui n'a reculé que de 2 à $3 \mathrm{~m}$.

Toutes les périodes étudiées, antérieures ou postérieures à la tempête montrent une érosion récurrente de la zone d'étude. Entre décembre 2007 (après le rechargement) et décembre 2008 (quelques mois après la tempête), le volume global des pertes sédimentaires sur la plage des Éloux est supérieur à $17000 \mathrm{~m}^{3}$. L'érosion constatée autour de la tempête (voir plus haut) est aussi observée en dehors de cet épisode puisque entre mars 2008 et mars de l'année suivante, les volumes perdus sont supérieurs à $18000 \mathrm{~m}^{3}$. De même, si l'on compare les périodes de décembre 2007mars 2008 à celles de décembre 2008-mars 2009, on constate que les volumes perdus sont presque équivalents ( $569 \mathrm{~m}^{3}$ de différence), (fig. 6).

En revanche, si entre juin 2006 et mars 2008, la tendance est plutôt à l'accrétion $\left(>27000 \mathrm{~m}^{3}\right)$, ces résultats sont faussés par le rechargement de plus de $51000 \mathrm{~m}^{3}$ qui a eu lieu entre décembre 2006 et avril 2007. En réalité, extrapolés, ces chiffres révèlent, pour la plage, une perte de plus de $20000 \mathrm{~m}^{3}$.

Enfin, sur une période de trois années écoulées (entre juin 2006 et mars 2009) et un rechargement effectué, le bilan est positif, mais de seulement $8700 \mathrm{~m}^{3}$, ce qui signifie qu'il y a eu une perte nette de plus de $45000 \mathrm{~m}^{3}$ de sédiments. 


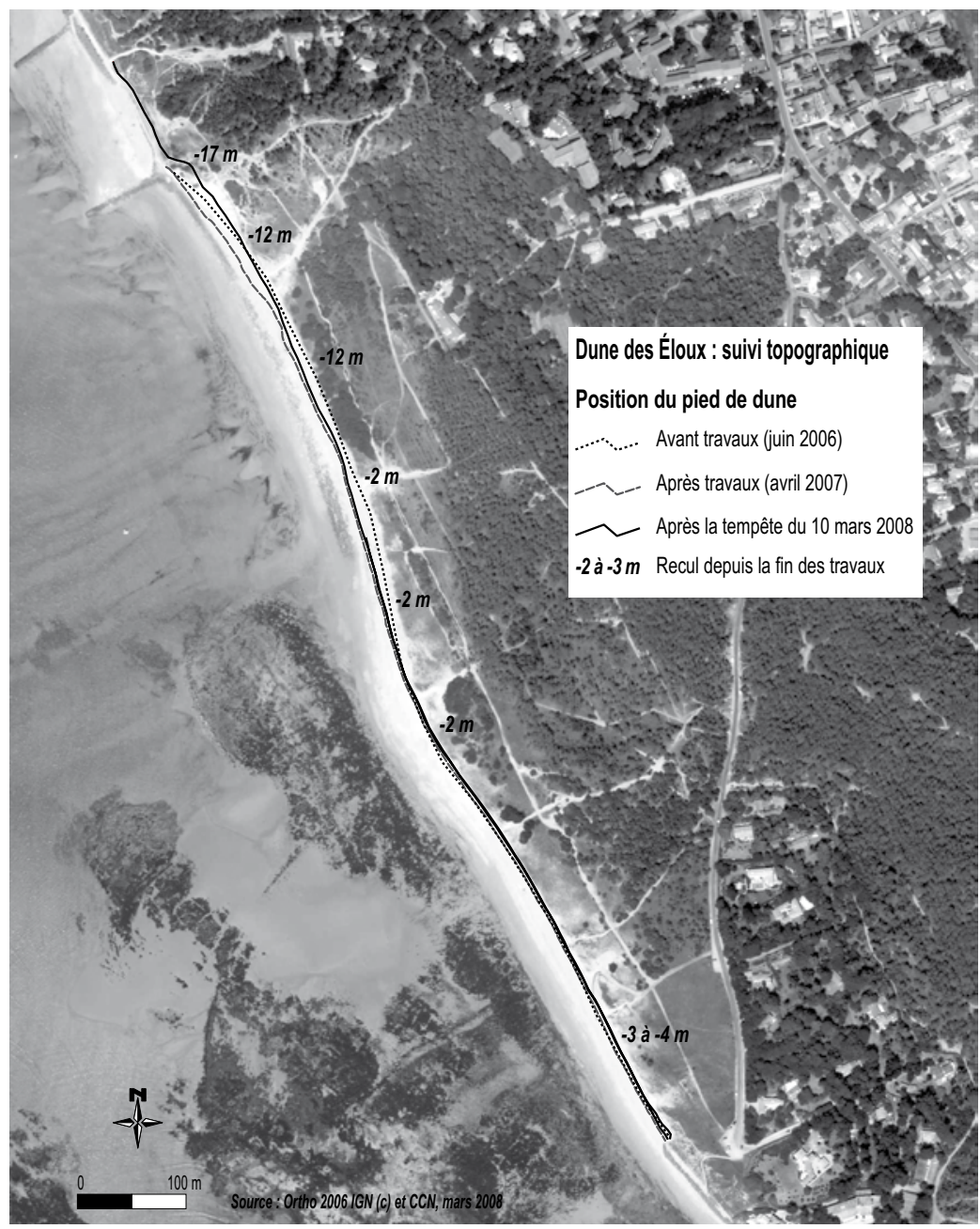

Figure 5 : Variations de la position du pied de dune (entre juin 2006 et mars 2008)

Shift of
dume / beach limit between june 2006 and march 2008

Les pertes enregistrées sur la plage des Éloux, entre 1999 et 2007, sont de loin les plus importantes de toute la façade ouest de l'île et sont supérieures à $100000 \mathrm{~m}^{3}$ (CCN, 2008). Plus au Nord du secteur d'étude, la plage de L'Épine enregistre, pour la même période, les secondes pertes les plus importantes avec des volumes proches de $69000 \mathrm{~m}^{3}$ (tableau 2).

Au total, si l'on procède à un bilan entre la pointe du Devin et la pointe de la Loire, pour une période allant de 1999 à 2007 et que l'on tienne compte des gains de la plage du Morin $\left(89000 \mathrm{~m}^{3}\right)$, le volume globalement perdu est de l'ordre de $83000 \mathrm{~m}^{3}$ (CCN, 2008). Si l'on replace ce constat dans le contexte du plus long terme à la lumière des témoins géomorphologiques comme évoqués dans le cadre morphologique général (Ters, 1961), le glissement vers l'est du système dune/plage semble inéluctable et se fait par à-coups à la faveur (i) d'une érosion ordinaire ponctuée d'évènements pouvant l'accélérer lorsque les conditions météo-marines sont toutes favorables, ou (ii) d'évènements certes puissants mais où la combinaison des éléments n'est pas totalement favorable à l'expression d'un recul temporairement plus rapide, ce qui est le cas de la tempête de mars 2008.

Les cartes de cubatures réalisées entre avril 2007 et mars 2008 (après les travaux de mise en place des pieux) puis entre décembre 2007 et mars 2008 (avant-après tempête) (fig. 7 - 


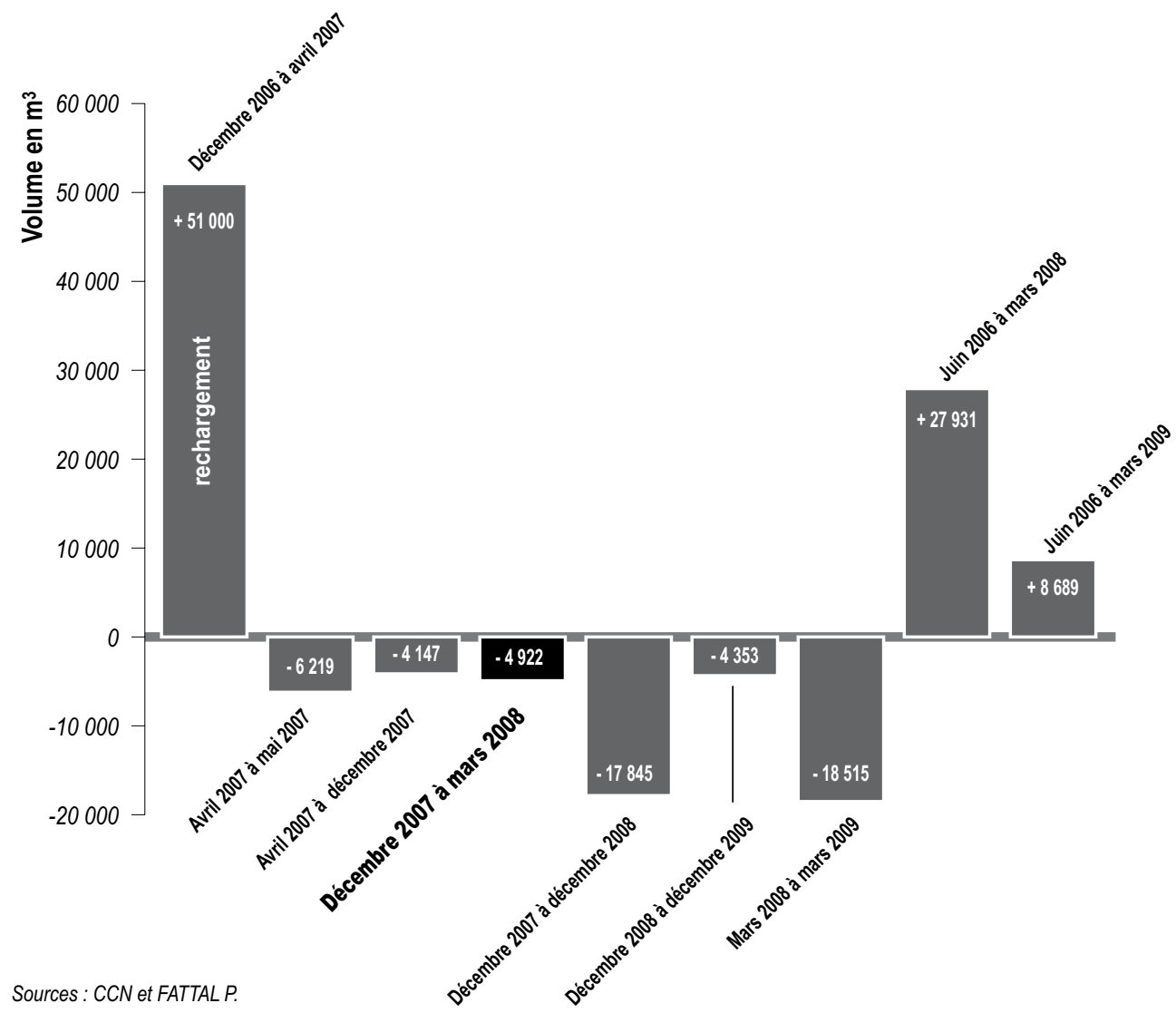

Figure 6 : Bilans sédimentaires pour le secteur des Éloux Les Éloux beach and dune sediment budget

planche VI) montrent que c'est le haut de plage qui est principalement touché par l'érosion. Ces deux cartes sont aussi intéressantes car entre les deux périodes, 11 mois pour la première et 3 à 4 mois pour la seconde qui est un sous-ensemble de la première, on constate que les zones en accrétion ont migré vers le sud (approximativement $28000 \mathrm{~m}^{3}$ selon la CCN, 2009) et cette observation est conforme au transit sédimentaire observé sur cette façade de lî̂le (Safege-Cetis, 2003; DHI, 2008).

\section{Discussion}

Au total, l'effet constaté de la tempête du 10 mars 2008 n'est pas proportionnel au potentiel morphogénique de cet évènement. En dépit de la conjonction de la tempête, des forts coefficients de marée enregistrés ce jour-là et de la coïncidence à l'heure près de la haute mer du matin avec la dépression barométrique maximale, trois éléments d'explications pourraient être avancés qui concourent à minimiser les effets morphogéniques. Ces éléments restent du domaine de l'interprétation en l'absence de mesures in situ avant et après tempête à la fois sur la plage et sur l'avant-plage. Le premier, tient au fait que dans le secteur et pour le jour considéré, la dérive a été inverse, due aux houles de sud-ouest exerçant probablement des transferts sédimentaires pou- 
vant localement cicatriser les formes affectées par la tendance générale aux transferts vers le sud. Cette dérive inverse à d'ailleurs été modélisée par le bureau d'études Safege-Cetis (2003) pour des houles incidentes de sud-ouest $(2 \mathrm{~m}-8 \mathrm{~s}$ et $5 \mathrm{~m}$-10 s). Un effet de compensation aurait pour résultante une minimisation des effets d'érosion constatés. En second lieu surtout, les sédiments piégés sur la plateforme d'abrasion de la pointe de la Loire, résultante des dérives générales, ont pu être partiellement remobilisés et redistribués sur le secteur des Éloux, concrétisant un apport sédimentaire minimisant l'effet érosif. Un levé topographique de la zone intertidale réalisé en 2006 montre l'existence d'un dépôt sédimentaire orienté ouest-est d'environ $800 \mathrm{~m}$ de long pour $200 \mathrm{~m}$ de large et d'une épaisseur moyenne de $0,5 \mathrm{~m}$ (soit environ $80000 \mathrm{~m}^{3}$ ), doublé d'un second dépôt plus au sud (fig. 8 - planche VII). Ces dépôts, alimentés par les dérives générales pourraient servir, en cas d'inversion, à maintenir voire engraisser la plage vers le nord.

Enfin, et c'est ce qui rend complexe l'interprétation des résultats dans le secteur, la présence des nombreux ouvrages (épis, perré bas, pieux hydrauliques et ganivelles sur la dune) ont eux aussi probablement eu un effet réducteur de l'érosion. Ces divers éléments sont donc de nature à moduler l'effet de cette tempête.

\section{Conclusion}

L'analyse historique a permis la reconnaissance du système côtier dans son état naturel et le suivi de sa transformation en fonction des aménagements successifs. L'étude diachronique, basée sur les photographies aériennes des missions de 1950, 1974, 2000 et 2006 a permis la quantification de l'érosion linéaire sur une période de 56 ans. Il en ressort que pour la période de 1999 à 2006, le taux moyen de recul du trait de côte est de 1,9 m/an. L'érosion observée au moment de la tempête de mars 2008 n'est qu'un épisode parmi d'autres, dans un contexte plus général d'érosion chronique. Le secteur à bénéficié de conditions particulières le 10 mars 2008 avec des directions de vents et des houles associées qui ont généré une dérive inversée minimisant l'effet tempête et la tendance érosive naturelle de la plage. De plus, la présence de la résultante des dérives générales sur la plateforme avant-littorale sous la forme de stocks sédimentaires est probablement la clé de compréhension du fait que la tempête de mars 2008 n'a pas entraîné d'effets dévastateurs au niveau du secteur des Éloux.

Cependant, il faut rappeler que les pertes enregistrées sur la plage des Éloux restent malgré tout de loin les plus importantes de toute la façade ouest de l'île, puisqu'elles ont été supérieures à $100000 \mathrm{~m}^{3}$ pour la période comprise entre 1999 et 2007. Ceci s’inscrit dans la suite logique d'un déplacement longitudinal plus général et sur le long terme du dispositif plage/dune vers l'est.

\section{Remerciements}

Cette recherche s'intègre dans le programme de recherche ANR $n^{\circ}$ ANR-06-VMC-009), VULSACO (VULnerability of SAndy COast systems to climatic and anthropic changes). Les auteurs remercient tous les organismes publics pour les données utilisées dans cet article (CETMEF, Météo-France SHOM et Communauté de Communes de l'île de Noirmoutier). 


\section{Références}

Battiau-Queney Y., Billet J.-F., Chaverot S., Lanoy-Ratel P., 2003. Recent shoreline mobility and geomorphologic evolution of macrotidal sandy beaches in the north of France, Marine Geology, vol. 194, $n^{\circ} 1-2$, p. 31-45.

Bouligand R., Pirazzoli P. A., 1999. Les surcotes et les décotes marines à Brest, étude statistique et évolution, Oceanologica Acta, 22, p. 153-166.

Caspar R., Costa S., Jаков E., 2007. Fronts froids et submersions de tempête dans le nord-ouest de la France Le cas des inondations par la mer entre l'estuaire de la Seine et la baie de Somme, La Météorologie, $\mathrm{n}^{\circ} 57$, p. 37-47.

CCN, 2000. Programme pluriannuel de sécurité des populations face à la mer de l'île de Noirmoutier, Bulletin spécial du District, 16 p.

—, 2004. Dune des Eloux, fiche de suivi individuel, document de l'observatoire du littoral, planche cartographique, (non publié).

—, 2008. Évolution des plages par transit - période 1999-2007, document de l'observatoire du littoral, planche cartographique, (non publié).

—, 2009. Île de Noirmoutier, site des Eloux, suivi des travaux expérimentaux de pieux hydrauliques, planche cartographique, (non publié).

DHI, 2008. Etude de connaissance des phénomènes d'érosion sur le littoral vendéen. Étude détaillée de la cellule $n^{\circ} 2$ : «Côte Ouest de l'Île de Noirmoutier », Rapport pour la DDE 85, 90 p., (non publié).

Fattal P., Maanan M., Tillier I., Rollo N., Robin M., Pottier P. Coastal vulnerability to oil spill pollution : the case of Noirmoutier Island (France), Journal of Coastal Research, sous presse.

Folk R. L., 1966, A review of grain-size parameters, Sedimentology, vol. 6, p. 73-93.

Gautier F., 1972. Phénomènes littoranx et protection des rivages sur le pourtour de la baie de Bourgneuf, Thèse de $3^{\mathrm{e}}$ cycle, Université de Rennes, 562 p.

Gautier M., 1979. Les effets des tempêtes de fin décembre 1978 et du premier janvier 1979 sur les côtes de Noirmoutier (Vendée) et du Pays de Retz (Loire-Atlantique), Norois, n 103, p. 369-382.

Gourlay M. R., 1992. Wave set-up, wave run-up and beach water table : Interaction between surf zone hydraulics and groundwater hydraulics, Coastal Engineering, vol. 17, p. 93-144.

Greenwood B., Osborne P. D., 1990. Vertical and horizontal structure in cross-shore flows : An example of undertow and wave set-up on a barred beach, Coastal Engineering, vol. 14, p. 543-580.

Longuet-Higgins M. S., Stewart R. W., 1964. Radiation stresses in water waves : a physical discussion, with applications, Deep-Sea Research, vol. 11, p. 529-562.

Morzadec-Kerfourn M. T., 1995. Coastline changes in the Armorican Massif (France) during the Holocene, Journal of Coastal Research, vol. 17, p. 197-203.

Pirazzoli P. A., Costa S., Dornbusch U., Tomasin A., 2006. Recent evolution of surge-related events and assessment of coastal flooding risk on the eastern coasts of the English Channel, Ocean Dynamics, vol. 56, p. 498-512.

Pirazzoli P. A., Costa S., Dombush F., 2007. Flood threat anomaly for the low coastal areas of the English Channel based on analysis of recent characteristic flood occurrences, Ocean Dynamics, vol. 57, p. 501510.

Regnauld H., Pirazzoli P. A., Morvan G., Ruz M., 2004. Impacts of storms and evolution of the coastline in western France, Marine Geology, vol. 210, n 1-4, p. 325-337.

Robin M., 2002. Télédétection et modélisation du trait de côte et de sa cinématique. Dans Le littoral : regards, pratiques et savoirs, Éditions Rue-d’Ulm /Presses de l'École normale supérieure, Paris, 230 p.

SAFEGE-CETIS, 2003. Étude hydro-sédimentaire de la côte ouest de Noirmoutier, Rapport final provisoire, Communauté de Communes de l'île de Noirmoutier, (non publié).

Sabatier F., Anthony E. J., Hequette A., Suanez S., Musereau J., Ruz M.-H., Regnauld H., 2009. Morphodynamics of beach/dune systems : examples from the coast of France, Géomorphologie : relief, processus, environnement, $\mathrm{n}^{\circ} 1$, p. 3-22.

Svendsen I. A., 1984. Wave heights and set-up in a surf zone, Coastal Engineering, 8, p. 303-329. 
Swift R. H., 1993. A simplified model for wave height and set-up in the surf zone, Coastal Engineering, vol. 19, p. 189-206.

Ters M., 1961, La Vendée littorale, étude de géomorphologie, Thèse d'état, Faculté des Lettres, Rennes, Imp. Oberthür, 578 p.

Tonnerre M.-A., 2001. Contribution à l'étude des tempêtes dans la Manche et en façade Atlantique de la France, au nord de l'île de Noirmoutier (1965-1994), thèse, Université de Lille 1, 2 volumes, 363 et 109 p.

Wakelin S. L., Woodworth P. L., Flather R. A., Williams J. A., 2003. Sea-level dependance on the NAO over the NW European continental shelf, Geophysical Research Letters, vol. 30, p. 56-59.

Cet article a été reçu le 22 septembre 2009 et définitivement accepté le 30 avril 2010. 
planche VI (Paul FATTAL et al. - Effets des tempêtes sur une plage aménagée et à forte portection côtière...)

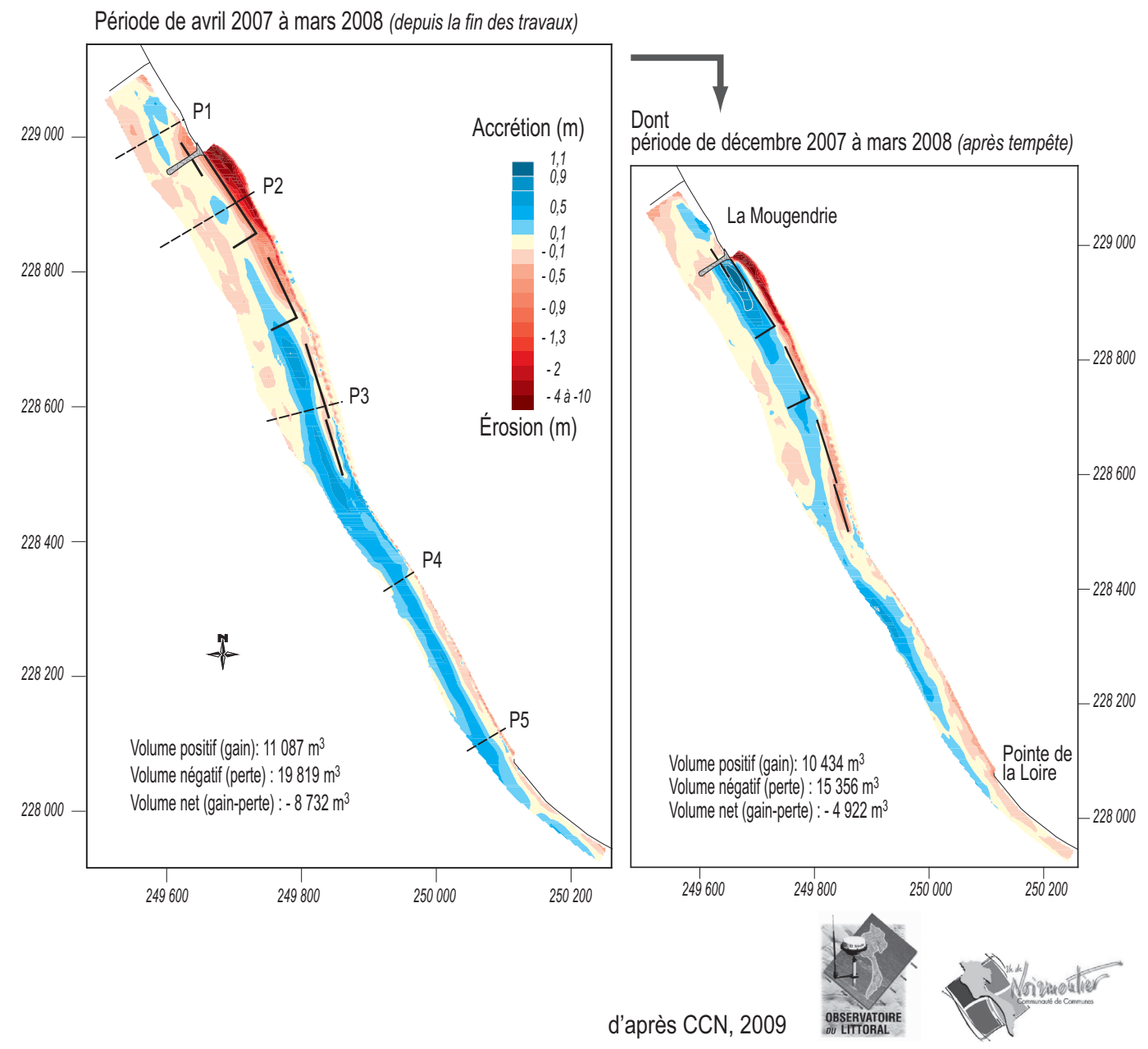

Figure 7 : Cubatures du site des Éloux avant-après tempête 2008

Les Éloux beach sediment budget before and after the 10 march 2008 storm 
planche VII (Paul FATTAL et al. - Effets des tempêtes sur une plage aménagée et à forte portection côtière...)

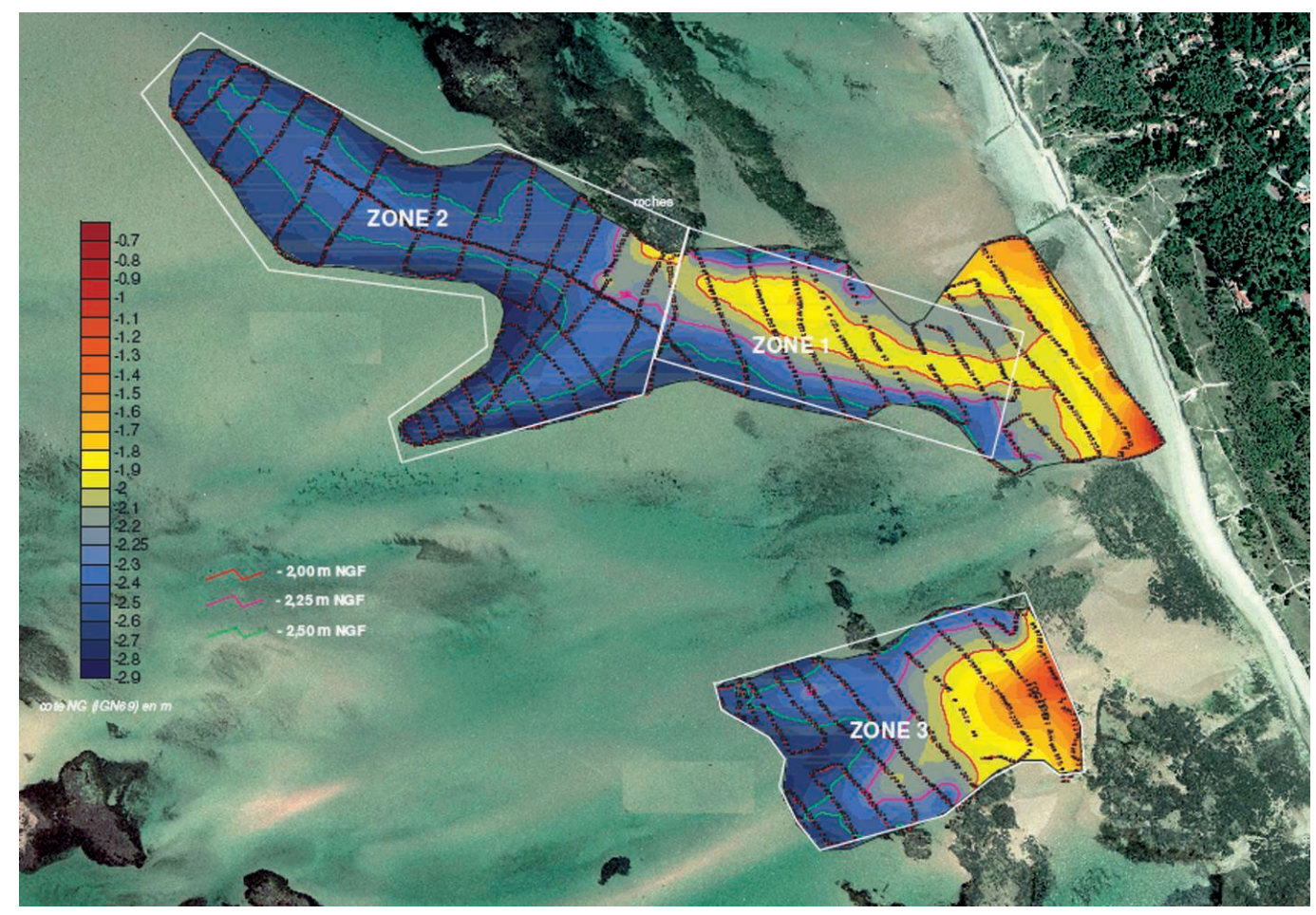

Figure 8 : Mise en évidence de dépôts sédimentaires en zone intertidale pouvant être remobilisés dans le contexte d'une tempête comme celle de mars 2008

Intertidal sand banks source likely to be solicited by major storms like March 2008 storm 